\title{
Changing plans: Dynamic inconsistency and the effect of experience on the reference point
}

\author{
RACHEL BARKAN and JEROME R. BUSEMEYER \\ Indiana University, Bloomington, Indiana
}

\begin{abstract}
This article presents a new test of a principle of decision making called dynamic consistency. This principle was tested in an experiment in which participants were asked to make decisions about a second gamble within a sequence of two gambles. Participants were first asked to make a planned choice about the second gamble. The planned choice was made before the first gamble was played and was conditioned on the anticipated outcomes of the first gamble. After the first gamble was played, the same participants were asked to make a final choice about the second gamble, conditioned on the experienced outcome of the first gamble. The results showed that participants' final choices were frequently inconsistent with their plans, even when the anticipated and experienced outcomes were identical. These inconsistencies occurred in a systematic direction. Experiencing an anticipated gain resulted with a change toward risk aversion, and experiencing an anticipated loss resulted in a change toward risk seeking. These results are explained in terms of the effect of actual experience on the reference point used for the evaluation of the decision problem.
\end{abstract}

Many real-life decisions involve multiple stages of choices and uncertain events. For example, imagine a patient who has to go through a laser operation to correct her eyesight. This procedure includes two surgeries that are done separately for each eye. Each surgery has a chance to either succeed or fail. Imagine further that one eye is so bad it has to be operated on, but that the second eye is in a better shape. The patient has to decide ahead of time (i.e., make a plan) whether she wants to have the second eye operated on or not, before experiencing the outcome of the first surgery.

\section{Dynamic Consistency}

The basic paradigm for analyzing decision problems of this kind utilizes a graphical representation called a decision tree (see Clemen, 1996; Keeny \& Raiffa, 1976; Raiffa, 1968; von Winterfeldt \& Edwards, 1986). Figure 1 presents a decision tree for the eye surgery example. Each surgery is represented as a chance event (with a circle) resulting in either success or failure. A success occurs with probability $P$, and provides the patient with some positive outcome. A failure occurs with probability $1-P$, and provides the patient with some negative outcome. The squares in Figure 1 represent decision nodes. Decision Node 1 represents the choice of having/not having the second surgery if the first one succeeds. Decision Node 2 represents the same choice conditioned on a failure of the first surgery.

This research was supported by federal research grants NIMH Perception and Cognition Grant R01 MH55680 and NSF Decision Risk Management Science Grant SBR-9602102. Correspondence should be addressed to J. R. Busemeyer, Department of Psychology, 1101 E. 10th St., Indiana University, Bloomington, IN 47405 (e-mail: jbusemey@) indiana.edu).
The generally prescribed procedure for planning a path through the tree is called backward induction (see Clemen, 1996; Keeny \& Raiffa, 1976; Raiffa, 1968; von Winterfeldt \& Edwards, 1986). Backward induction requires the decision maker to plan a path that reflects his/ her preferences, conditioned on anticipated future chance events. In the eye surgery example, a plan could be summarized with if-then statements such as: "If the first surgery succeeds/fails then a second surgery will/will not be taken." The procedure of backward induction is based on a fundamental principle of decision making called $d y$ namic consistency (see Machina, 1989; Sarin \& Wakker, in press). To obey this principle, the decision maker is obligated to carry out the plan if he/she actually experiences the anticipated outcomes on which the plan was based. The purpose of this paper is to empirically test this principle.

Dynamic consistency can be illustrated by referring to the example shown in Figure 1. Suppose the patient in the example plans to have the second surgery if the first one succeeds, but she plans not to have it if the first one fails. After experiencing a success, a dynamically consistent patient would follow through with the plan and decide to have the second surgery. Alternatively, after experiencing a failure, the dynamically consistent patient would follow through with the plan by not having the second surgery. However, it is not difficult to think of scenarios where the planned preference may change after having the first surgery. For example, if the first surgery is successful, the patient may be satisfied with the improvement in her eyesight, and change her original plan by not having the second surgery. Alternatively, if the first surgery fails, she might feel greater need to improve her eyesight, and change her original plan by having the operation on the second eye. Both of these changes in 


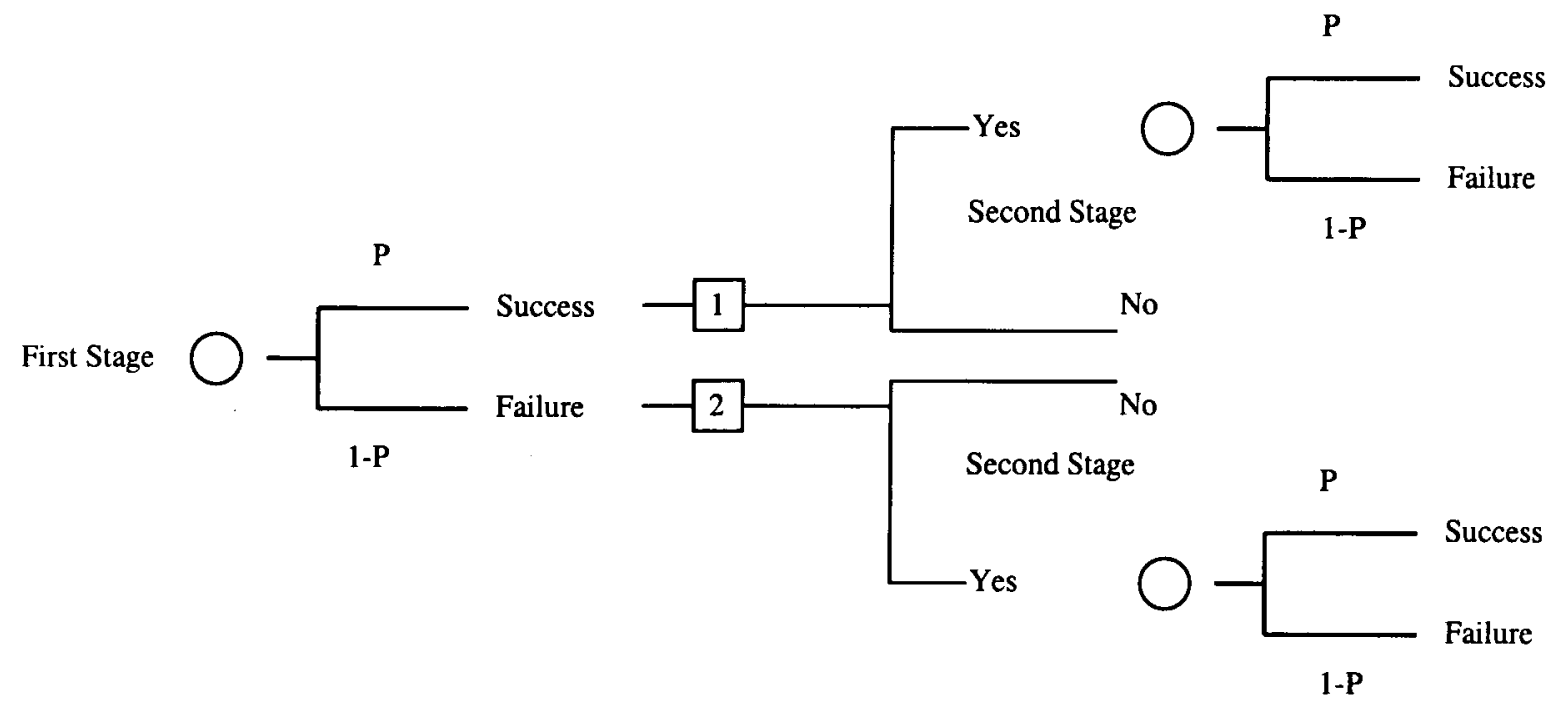

Figure 1. A decision tree representing the eye surgery example. Each surgery is represented as a chance event (with a circle), resulting in success or failure. Two decision nodes (squares) represent the choice between having or not having the second surgery, conditioned on the outcome of the first one.

preferences following experience with the planned outcomes violate dynamic consistency, calling into question one of the basic assumptions underlying decision analysis (see also Busemeyer, Weg, Barkan, \& Li, 1998).

\section{Sequential Gambling Paradigm}

Realistic decisions, like the eye surgery example, are too complex for providing uncontested tests of dynamic consistency. Instead, we employed a simple modification of this example, called the sequential gambling paradigm, where the chance nodes represent monetary gambles rather than surgical outcomes. The sequential gambling paradigm was previously used by Tversky and Shafir (1992) to test another principle of decision making called the "sure thing" principle: If Gamble A is preferred to Gamble B under every possible state of nature, then A should be preferred to $\mathrm{B}$ without the person knowing the state of nature.

In Tversky and Shafir's (1992) experiments, participants were told to imagine that they had just played a gamble that gave an equal chance to win $\$ 200$ or lose $\$ 100$. They had to decide whether or not they would play the same gamble a second time. When imagining they either won or lost the first gamble, most participants preferred to play the gamble once more. Yet, when participants were told to imagine that they did not know the result of the first gamble, most of them preferred not to play the second gamble. This change in preference was interpreted as a violation of the "sure thing" principle.

Although Tversky and Shafir (1992) restricted their paradigm to perform a test of the "sure thing" principle, it can easily be modified to provide a test of the dynamic consistency principle in the following way. In the present experiment, participants were presented with a sequence of two identical gambles, with the first one being oblig- atory (Figure 1). The participants were required to make two choices regarding the second gamble. A planned choice was made before they experienced the first gamble: Participants were asked whether they would take the second gamble if they won the first one, and whether they would take the second gamble if they lost the first one. Then a second final choice was made after they had experienced the actual outcome of the first gamble. If dynamic consistency holds, then the final choice should be identical to the planned choice. However, extending the results reported by Tversky and Shafir, we would suggest that this might not be the case, and that preferences may change once the result of the first gamble is experienced.

\section{Changing Reference Points}

Tversky and Shafir (1992) explained their violations of the "sure thing" principle in terms of a change in the reference point used to evaluate the outcomes. When participants were told to imagine they did not know the outcome of the first gamble, they evaluated the gains and losses of the second gamble with a neutral reference point of zero. In other words, they compared the gamble (win $\$ 200$ or lose $\$ 100$ ) with no gain or loss. However, when they were told to imagine, for example, losing $\$ 100$ from the first gamble, they evaluated the second gamble with respect to a new reference point that included this recent loss. In other words, they compared the redefined gamble (win $\$ 100$ or lose $\$ 200$ ) with a reference point equal to $-\$ 100$. Alternatively, when they were told to imagine, for example, winning $\$ 200$ from the first gamble, they evaluated the second gamble with respect to a new reference point that included this recent win. In other words, they compared the redefined gamble (win $\$ 400$ or win $\$ 100$ ) with a reference point equal to $\$ 200$. 
If participants change their reference point only after experiencing the outcomes of their plan, then systematic violations of dynamic consistency are expected to occur as follows. Consider an analysis based on the standard Sshaped utility function displayed in the left panel of Figure 2 (see Tversky \& Shafir, 1992). During planning, the expected utility ${ }^{2}$ of the second gamble (winning $\$ 200$ or losing $\$ 100$ ) is greater than the reference point of zero, and thus the second gamble is accepted. After experiencing a gain of $\$ 200$ from the first gamble, the expected utility of the second gamble (winning $\$ 400$ or winning $\$ 100$ ) is less than the utility of the gain from the first gamble, and the gamble is rejected. Thus, a person with a utility curve shown in Figure 2 would change from planning to take the gamble to choosing not to take the gamble after experiencing a gain, thus violating dynamic consistency.

More generally, we hypothesize that changes in the reference point will create dynamic inconsistencies in systematic directions. Experiencing a gain moves the reference point away from the linear toward the more concave part of the value function, producing more risk aversion. A second gamble, reevaluated with respect to this new reference point, would seem less attractive than before this gain took place. Participants who initially plan to play the gamble may change after this experience and decide to not play. For a similar reason, experiencing a loss moves the reference point away from the linear toward the more convex part of the value function, producing more risk seeking. A second gamble, reevaluated with respect to this new reference point, would seem more attractive than before this loss took place. Participants who initially plan not to play the gamble may change after this experience and decide to play.

\section{METHOD}

The following experiment was run to test the hypothesis just described. Using the sequential gambling paradigm with the changes noted earlier, the participants were presented with 32 decision problems. Each decision problem consisted of a sequence of two identical gambles, with the first gamble being obligatory. For each decision problem, participants made two choices. The first choice occurred before the first gamble took place, and participants were asked to decide whether or not they would take the second gamble, conditioned on the possible outcome of the first gamble. The second choice occurred after the result of the first gamble was experienced, and the participants were asked to make a final choice regarding the second gamble. In order to make both choices equally important to the decision maker, the computer randomly chose one of these two choices (planned choice or final choice) with equal probabilities and delivered real monetary gains or losses accordingly. For example, if the decision maker planned to take the second gamble, but then changed his/her mind and decided not to gamble, and the computer randomly selected the planned choice, then the second gamble would be played out to determine the payoff for that trial. The critical measure was the consistency between the first choice (representing the plan) and the final choice, conditioned on the outcome of the first gamble.

\section{Participants}

One hundred and fifty students taking an introductory psychology course at Indiana University participated in the experiment. They were recruited by advertisements offering one credit for the course. They also earned a monetary reward of $\$ 2-\$ 8$ according to the number of points earned by gambling, where each point in the experiment was worth $\$ .25$. The average payoff was $\$ 5.30$.

\section{Design}

The basic set of decision problems consisted of four pairs of identical gambles. One gamble \{win 200 points or lose 100 points with $50 \%$ used the same numerical values as used by Tversky and Shafir (1992). A second gamble \{win 80 or lose -40 with $50 \%$ \} produced the same ratio with different values. The remaining gam-

\section{Example Utility Curve}

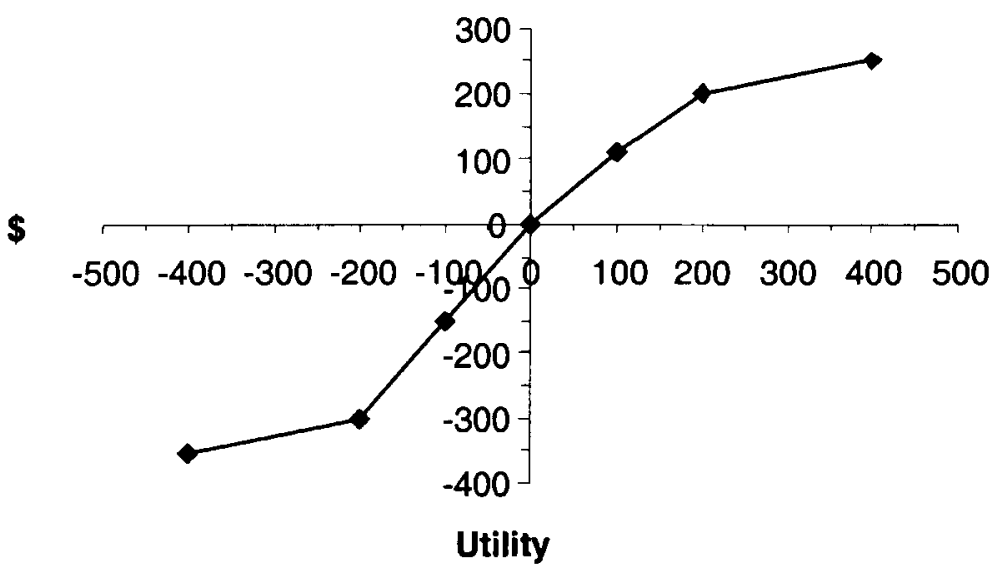

Figure 2. A hypothetical S-shaped value function. Close to the origin the function is almost linear. Further from the origin the function becomes concave for gains and convex for losses. Experiencing a gain moves the reference point toward the concave part of the function, creating risk aversion. Experiencing a loss moves the reference point toward the convex part of the function, creating risk seeking. 
bles $\{$ win 200 or lose -40 with $50 \%$ \} and \{ win 80 or lose -100 with $50 \%$ \} produced the more and less attractive gambles, respectively. Controlling for the outcome of the first gamble, and for the outcome of the second gamble, each decision problem was presented with all the possible outcome combinations (i.e., win-win, win-lose, lose- win, lose-lose). The 16 decision problems (four gambles $X$ four outcomes combinations) were replicated twice. The order of presentation was counterbalanced.

The present experiment was also designed to extend the Tversky and Shafir (1992) results ${ }^{3}$ by examining three types of plan scopes. One third of the participants were always asked to make a general plan that did not focus attention on the outcome of the first gamble. With this plan, participants were simply asked: "Will you take the second gamble or not?" This corresponds to Tversky and Shafir's condition where they asked participants to decide about the second gamble without knowing the outcome of the first gamble.

Another third of the participants were asked to make an elaborated plan conditional on both winning and losing possibilities With this plan participants had to answer two questions: "Suppose you win the first gamble; will you take the second gamble or not?" and, "Suppose you lose the first gamble; will you take the second gamble or not?" This corresponds to Tversky and Shafir's (1992) condition where they asked participants to decide about the second gamble after imagining they knew the outcome of the first gamble.

The remaining third of the participants had to make partial plans exclusively for the possibility of winning or exclusively for the possibility of losing the first gamble (but not both). For eight presentations of each basic gamble, participants were asked: "Suppose you win the first gamble; will you take the second gamble or not?" For the other eight presentations of each basic gamble, participants were asked: "Suppose you lose the first gamble; will you take the second gamble or not?" Half the time, the partial plan was relevant (i.e., plan for winning the first gamble and the first gamble was won, or plan for losing the first gamble and the first gamble was lost). Half the time, the partial plan was irrelevant (i.e., plan for winning the first gamble and the first gamble was lost, or plan for losing the first gamble and the first gamble was won).

\section{Procedure}

Instructions were presented on a computer monitor, and for each decision problem, participants were presented with a sequence of on-screen dialogues. Note that they did not actually see any decision trees such as the one shown in Figure 1. Instead, all the information was presented textually, Responses were made using the mouse to choose between possible options (e.g., accepting or rejecting the second gamble). The sequence of the on-screen dialogues for each decision problem allowed participants to (1) review the values and probabilities of the two gambles; (2) make a plan for the second gamble; (3) experience the first gamble and its result; and (4) make a second decision regarding the second gamble. At this point one of the two decisions was sampled at random to determine whether the second gamble would take place or not. Each trial ended with a summary of the total earnings in the specific decision problem. The first decision problem served as practice to make sure that the participants understood the task. This practice problem was followed with 32 decision problems. Four of the latter 32 trials were sampled at random to determine the final take-home pay for each participant.

The experiment was computerized and lasted $30 \mathrm{~min}$. It was programmed using Visual Basic 3 for Windows, and presented on a Pentium PC with a Super VGA 16-in. screen.

\section{RESULTS}

The results are presented in two parts. First we present the basic results concerning the proportion of trials in which the second gamble was chosen for each condition, and these results are compared with Tversky and Shafir's (1992) findings. Next we present the new critical results showing the rates of dynamic inconsistency for each of the conditions.

\section{Choice Proportions}

The cells in Table 1 present the proportion of trials in which the second gamble was chosen under each of the conditions. The first column shows the payoff condition corresponding to each row; columns $2-4$ represent the general plan, columns 5-8 represent the elaborated plan, and columns 9-12 represent the partial plan. Within the general plan, the first column shows the planned choices (independent of anticipated outcome), and the next two columns show the final choices conditioned on the actually experienced win or loss. Within the elaborated and partial plans, the first pair of columns shows the choices conditioned on an anticipated or actually experienced win, and the second pair of columns shows the choices conditioned on anticipated or actually experienced loss.

The frequency of choosing the gamble increased down the rows as the winning payoff increased and as the losing payoff decreased in magnitude. However, there was almost no effect of the plan scope on the frequency with which the gamble was chosen. For example, participants planned to choose the $\{200,-100\}$ gamble around $70 \%$

Table 1

Probability of Accepting the Second Gamble, Conditioned on the Anticipated or the Actual Outcome of the First Gamble, by the Four Gambles and Three Plan Scopes

\begin{tabular}{|c|c|c|c|c|c|c|c|c|c|c|c|}
\hline \multirow[b]{3}{*}{ Gamble } & \multicolumn{3}{|c|}{ General Plan } & \multicolumn{4}{|c|}{ Elaborated Plan } & \multicolumn{4}{|c|}{ Partial Plan } \\
\hline & \multirow{2}{*}{$\overline{\overline{\text { Ant. }}}$} & \multirow{2}{*}{$\frac{\text { Win }}{\text { Act. }}$} & \multirow{2}{*}{$\frac{\text { Lose }}{\text { Act. }}$} & \multicolumn{2}{|c|}{ Win } & \multicolumn{2}{|c|}{ Lose } & \multicolumn{2}{|c|}{ Win } & \multicolumn{2}{|c|}{ Lose } \\
\hline & & & & Ant. & Act. & Ant. & Act. & Ant. & Act. & Ant. & Act. \\
\hline $80,-100 ; .50$ & .23 & .22 & .32 & .26 & .24 & .33 & .42 & .23 & .16 & .19 & .27 \\
\hline $80,-40 ; .50$ & .73 & .63 & .74 & .76 & .68 & .68 & .70 & .76 & .70 & .75 & .76 \\
\hline $200,-100 ; .50$ & .72 & .53 & .80 & .70 & .60 & .68 & .73 & .66 & .59 & .68 & .76 \\
\hline $200,-40 ; .50$ & .82 & .72 & .91 & .84 & .77 & .85 & .87 & .83 & .74 & .86 & .90 \\
\hline
\end{tabular}

Note-Ant., anticipated outcome before the first gamble is played. Act., actual outcome experienced after the first gamble was played. For the general plan, there is only one column under Ant. because in this case participants were forced to make the same plan for both anticipated outcomes. The proportions within each cell were based on 200 observations. 
of the trials under all the planning conditions. It seems that participants simply ignored the anticipated outcomes when forming their plans. An interesting finding is that the frequency of choosing the gamble decreased following the actual experience of a win, and this frequency increased following the actual experience of a loss. For example, consider the $\{200,-100\}$ gamble for the elaborated plan condition: The frequency of choosing the gamble decreased from $70 \%$ to $60 \%$ following a win, but it increased from $68 \%$ to $73 \%$ following a loss. The direction of this change was consistent across payoff and planning conditions, and it is in accord with the changing reference point hypothesis. A more detailed analysis of this effect is reported in the section on rates of dynamic inconsistency. Before turning to the latter section, we need to compare the present results with Tversky and Shafir's (1992) findings.

Recall that Tversky and Shafir (1992) reported a strong preference not to play the $\{\$ 200,-\$ 100\}$ gamble a second time when the outcome of the first play was unknown. Contrary to this result, we found that the majority of participants preferred to play the gamble under all plans, including the general plan. Playing the gamble is a sensible option because the expected payoff $(200-$ $100) / 2=50$ is quite large. Apparently, the participants in Tversky and Shafir's study were much more risk averse than our participants. The reduced level of risk aversion exhibited by our participants may have resulted from one of the procedural differences mentioned in note 3 .

\section{Dynamic Consistency}

A dynamically inconsistent trial was recorded when a participant's planned choice differed from his/her final choice. For the elaborated and partial plan scopes, we considered the cases in which the anticipated and actual outcome of the first gamble were identical (for the partial plan, this resulted in reducing the number of tests by half). An example of a dynamically inconsistent trial for these plan scopes would be the following sequence: a planned choice not to take the second gamble conditioned on anticipated loss of the first gamble; actual experience of loss; and a final choice to take the second gamble. Planned choices in the general plan scope were not conditioned on a specific outcome. Rather, participants were forced to make the same plan for both anticipated outcomes. For this plan scope, a trial was considered inconsistent (not necessarily dynamically so) if the planned and final choices differed. The overall proportion of dynamically inconsistent trials was $19.13 \%$. Broken down by plan scope, the proportions of dynamic inconsistency were $17.56 \%$ with the elaborated plan, $18.13 \%$ with the partial plan, and $21.69 \%$ with the general plan. Broken down by the four payoff conditions, the proportions of dynamic inconsistency were $22.00 \%$ for the gamble $\{200,-100\}, 21.50 \%$ for the gamble $\{80,-40\}, 17.20 \%$ for the gamble $\{80,-100\}$, and $15.40 \%$ for the gamble
$\{200,-40\}$. However, our main concern is the direction of change.

Whenever a dynamically inconsistent trial occurred, it was also possible to measure the direction of the inconsistency. The direction of change is defined as if the first choice was not to take the second gamble, and the second choice was to take it. The direction of change is defined as conservative if the first choice was to take the second gamble and the second choice was not to take it. Considering the outcome of the first gamble (i.e., won or lost), there are four possible directions of inconsistency: (1) a risky change after experiencing a gain; (2) a risky change after experiencing a loss; (3) a conservative change after a gain; and (4) a conservative change after a loss.

Figures $3 \mathrm{a}-3 \mathrm{~d}$ present the relative frequencies of the four directions of inconsistency (given that a dynamically inconsistent trial occurred). Each panel represents a different payoff condition (i.e., gamble), each group of three bars represents one of the four possible directions of inconsistency, and each bar within a group represents one of the three plan scope conditions. There was almost no effect of plan scope, and therefore we collapsed the data across this factor to produce Figure $3 \mathrm{e}$. In this figure, each group of four bars represents one payoff condition, and each bar represents one of the four directions for change.

The findings presented in Figure 3 are in accordance with the changing reference point hypothesis: A risky change tended to occur after the experience of a loss, and a conservative change tended to occur after the experience of a gain. Taken together, these two directions composed almost $70 \%$ of the cases of dynamic inconsistency. There is an indication for an interaction between the four directions of inconsistency and the gambles (Figure 3 ). With the least preferred gamble $\{80,-100\}$, the most frequent direction of inconsistency was the risky change after a loss. With the most preferred gamble $\{200,-40\}$ and with the $\{200,-100\}$ gamble, the reverse happened, and the most frequent was the conservative change after a gain. The frequency of those two directions was almost equal with the $\{80,-40\}$ gamble.

\section{Dynamic Inconsistency or Choice Inconsistency}

We need to rule out the possibility that the results reflect choice inconsistency-that is, the tendency for people to change their minds when presented with the same choice twice. Table 2 shows the schematic combination of the two planned choices (accept/reject the second gamble) by the two final choices (accept/reject the second gamble). The top half of the table shows the actual outcome of winning the first gamble, and the bottom half shows the actual outcome of losing the first gamble. Consider first the top half of the table. The cells denoted $a$ and $d$ represent the cases of consistency, where the planned and final choices are identical. The cells denoted $b$ and $c$ represent the cases of inconsistency, where 
a

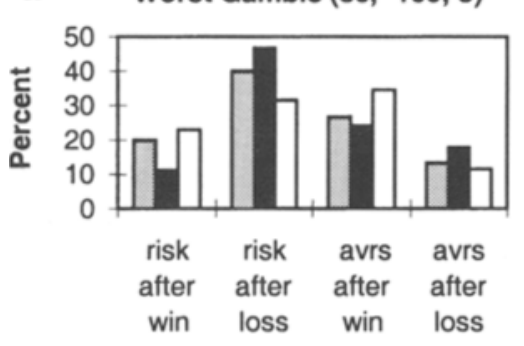

b

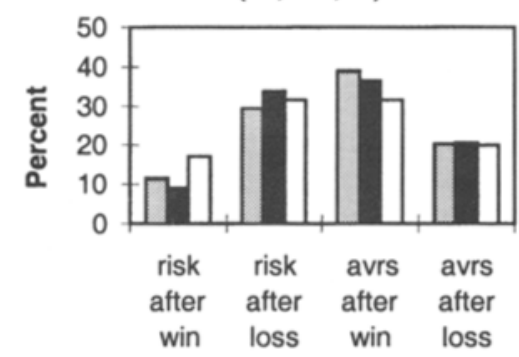

口general $\mathbf{E}$ elaborated Dpartial

c

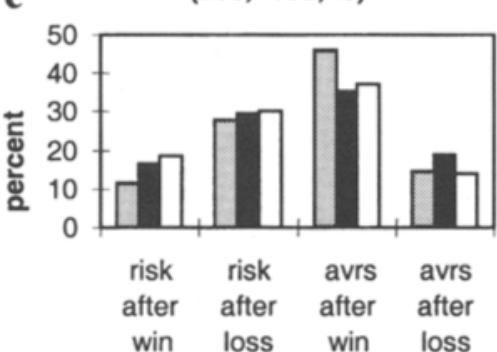

d Best Gamble $(200,-40 ; .5)$

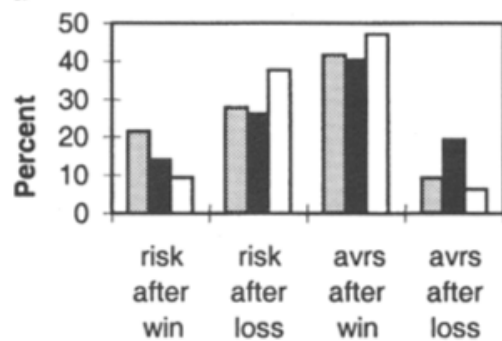

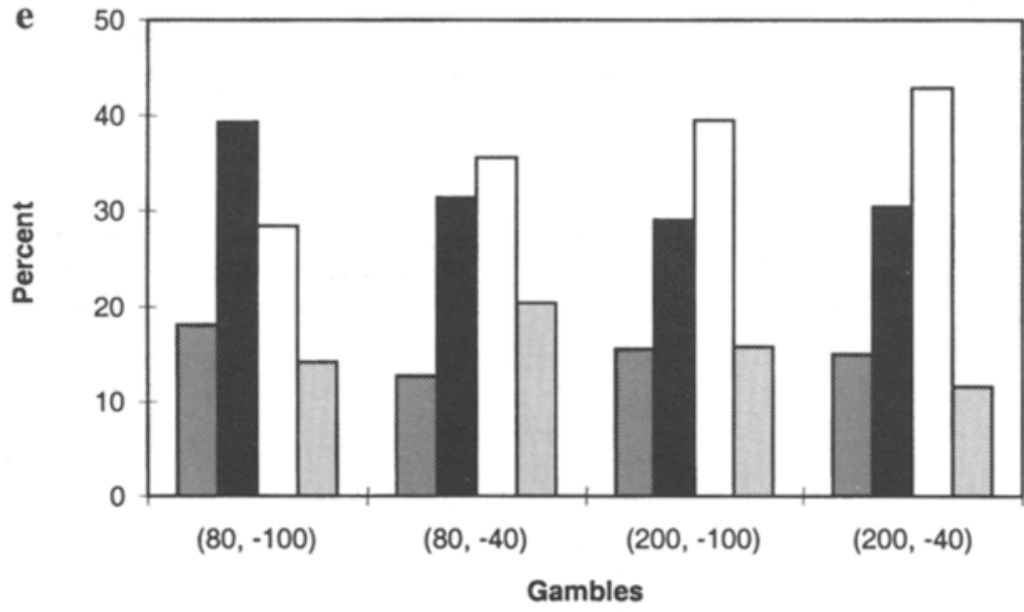

$\square$ risk after win $\square$ risk after loss $\square$ avrs after win $\square$ avrs after loss

Figure 3. Percent of the four directions of dynamic inconsistency (given that a dynamically inconsistent trial occurred). Figures 3a-3d present the directions for each gamble under each plan scope. Figure 3e collapses the data of the plan scopes and shows the general trend of directions by gambles.

the planned and final choices are different. If the inconsistencies observed in this research were the result of choice inconsistency, we would expect these cells to be identical, and the order of the choices should not matter $(b=c)$. The results shown in Figure 3 indicate that this was not the case. When the first gamble was won, the joint probability in cell $b$ was larger than the joint probability in cell $c$. The same argument could be made for the case where the first gamble was lost. The joined probabilities in cells $f$ and $g$ should have been identical.
The findings showed that when the first gamble was lost, the joint probability in cell $f$ was larger than the joint probability in cell $g$. Therefore, choice inconsistency alone cannot explain the systematic directions of dynamic inconsistency.

\section{Statistical Analysis}

A three-way analysis of variance (ANOVA) was performed with plan as a between-subjects factor and payoff and direction of inconsistency as repeated measures fac- 
Table 2

A Schematic Combination of the Two Planned Choices by the Two Final Choices for Winning and for Losing the First Gamble

\begin{tabular}{|c|c|c|c|}
\hline & & \multicolumn{2}{|c|}{ Final Choice } \\
\hline & & Accept & Reject \\
\hline \multicolumn{4}{|c|}{ Win the First Gamble } \\
\hline Planned & Accept & $a$ & $b$ \\
\hline Choice & Reject & $c$ & $d$ \\
\hline \multicolumn{4}{|c|}{ Lose the First Gamble } \\
\hline Planned & Accept & $e$ & $f$ \\
\hline Choice & Reject & $g$ & $h$ \\
\hline
\end{tabular}

tors. The dependent measure was the frequency of dynamically inconsistent trials. The results of this analysis revealed a main effect of payoff, showing that dynamic inconsistency was more frequent with the intermediate payoff conditions (gambles $\{200,-100\}$ and $\{80$, $-40\})$ than with the extreme payoff conditions (gambles $\{80,-100\}$ and $\{200,-40\})[F(3,441)=8.22, p<$ $.0001]$. The main effect of direction was also significant $[F(3,441)=38.47, p<.0001]$, indicating that a risky change after a loss on the first gamble and a conservative change after a win on the first gamble were the dominant directions of dynamic inconsistency. Finally, the interaction between payoff and direction (shown in Figure 3e and discussed above) was significant as well $[F(9,1323)=$ $2.15, p<.0001]$.

\section{DISCUSSION}

The main finding of this experiment was that dynamic inconsistencies occurred in a systematic direction: After the experience of a win, preferences tended to switch from taking the gamble toward rejecting it; after the experience of a loss, preferences tended to switch from rejecting toward taking the gamble. This is in accord with the changing reference point hypothesis: When planning, the decision maker evaluates the second gamble with a neutral reference point of zero. Experiencing a gain shifts the reference point toward the concave part of the utility function, and experiencing a loss shifts the reference point toward the convex part of the utility function.

An alternative explanation for the present findings is that experience with the outcomes of the first gamble triggered a change in the subjective probabilities, rather than the reference point. During planning, the decision maker may perceive the two gambles as independent of each other. In this case, the subjective probability of winning or losing the second gamble remains the same and does not depend on the anticipated outcome of the first gamble. But the actual experience of the outcome from the first gamble may trigger a "gambler's fallacy" type of reaction - experiencing a win causes the decision maker to expect a subsequent loss (i.e., the subjective probability of losing the second gamble increases). In a similar way, experiencing a loss causes the decision maker to expect a subsequent win (i.e., the subjective probability of winning the second gambles increases). These changes in subjective probability triggered by experience would cause the observed directions of dynamic inconsistency. However, this explanation fails to account for the interaction found between the direction of inconsistency and the gamble payoff. According to this explanation, all four directions of inconsistency should have occurred with the same frequency and should not have been affected by the payoff manipulation.

The change in the reference point hypothesis offers a simpler explanation for the interaction between gamble payoff and the inconsistency direction. A large gain should result with a larger change in the reference point than a small gain, and a large loss should result in a larger change in the reference point than a small loss. Larger shifts in the location of the reference point on the utility function produce greater changes toward risk aversion after gain and risk seeking after loss.

The idea that imagined preferences differ from experienced preferences is supported by other findings in the decision-making literature. For example, Kahneman and Snell (1992) found that the correlations between participants' predictions for their own change in taste and the actual change of taste were close to zero. Another example is provided by Loewenstein and Adler's (1995) investigation of the "endowment effect." They found that participants valued an object much less before owning than after actually owning it. Loewenstein and Adler suggested that the different values associated with that object were based on the different reference points of "not having" and "having" the object. The mood preservation hypothesis seems to be of a similar nature. Nygren, Isen, Taylor, and Dulin (1996) found that participants induced to have good mood tended to overestimate the chances of winning a gamble, but nevertheless, rejected it. It was suggested that participants were reluctant to risk the good mood they had gained earlier.

In conclusion, the principle of dynamic consistency implies that when we are given full information, actual experience should not change our planned preferences, and the utilities we use to evaluate the decision problem should stay constant. The present work suggests that experience results in a change of the reference point, and this change inevitably affects the perceived values associated with the next stage of the decision problem, making the original plan less desirable. Future research is needed to fully understand both the effect of actual experience and the dynamics of the reference point.

\section{REFERENCES}

Busemeyer, J. R., Weg, E., Barkan, R., \& Li, X. (1998). Dynamic and consequential consistencies of choices between paths within decision trees. Manuscript submitted for publication.

Clemen, R. T. (1996). Making hard decisions: An introduction to decision analysis. Boston: Duxbury Press.

Kahneman, D., \& SNell, J. (1992). Predicting a changing taste: Do people know what they will like? Journal of Behavioral Decision Making, 5, 187-200. 
KeENY, R. L., \& RAIFFA, H. (1976). Decisions with multiple objectives: Preferences and value tradeoffs. New York: Wiley.

Loewenstein, G., \& AdLER, D. (1995). A bias in the prediction of tastes. The Economic Journal, 105, 929-937.

MaChINA, M. J. (1989). Dynamic consistency and non-expected utility models of choice under uncertainty. Journal of Economic Literature, 27, 1622-1668.

Nygren, T. E., Isen, A. M., TAYLOR, P. J., \& Dulin, J. (1996). The influence of positive affect on the decision rule in risk situations: Focus on outcome (and especially avoidance of loss) rather than probability. Organizational Behavior \& Human Decision Processes, 66, 59-72.

RAIFFA, H. (1968). Decision analysis. London: Addison-Wesley.

SARIN, R., \& WAKKER, P. (in press). Consistency in dynamic choice situations with nonexpected utility models. Journal of Risk \& Uncertainty.

TVersky, A., \& Shafir, E. (1992). The disjunction effect in choice under uncertainty. Psychological Science, 3, 305-309.

VON WINTERFELDT, D., \& EDWARDS, W. (1986). Decision analysis and behavioral research. New York: Cambridge University Press.

\section{NOTES}

1. The sequential gambling paradigm is utilized as a tool. We do not wish to equate the "sure thing" principle with the dynamic consistency principle. The "sure thing" principle implies that planned choices may be the same even if they are based on different anticipated outcomes The principle of dynamic consistency does not put any restriction on the plan itself, but only demands that it would be carried out if the anticipated outcome (on which the plan is based) and the actual outcome are the same.

2. The utility function shown in Figure 2 is used for the following calculations. During the planning stage, the reference point is zero, so that the expected utility of the gamble is $[u(200)+u(-100)] / 2=$ $[200-150] / 2=25$, which is above the utility $u(0)=0$ for the sure thing. After the experience of a loss, the expected utility of the gamble is $[u(100)+u(-200)] / 2=[110-300] / 2=-95$, and again this is greater than the utility $u(-100)=-150$ of the loss; but after a win, the expected utility of the gamble is $[u(400)+u(100)] / 2=[250+$ $110] / 2=180$, which is less than the utility $u(200)=200$ of the gain.

3. Although we tried to extend the findings of Tversky and Shafir (1992), this study was not a replication for several reasons. First and foremost, we asked for planned choices before experience as well as for final choices after experience with the outcomes of the first gamble (as opposed to only asking for choices before the outcomes were actually experienced). Second, we used real monetary payoffs; the actual money earned by winning 200 points was $\$ .50$ and the actual money debited by losing 100 points was $\$ .25$ (as opposed to imagining a win of $\$ 200$ or a loss of $\$ 100$ ). Third, participants played 32 gambles in one session, 4 of which were randomly selected and played for real money at the end (as opposed to one hypothetical play).

(Manuscript received June 24, 1998; revision accepted for publication April 12, 1999.) 